\title{
Hernia femoral en niños: reto diagnóstico y terapéutico. Reporte de un caso
}

\author{
Femoral hernia in children: diagnostic and therapeutic challenge. Case report
}

Carlos García-Hernández*, Lourdes Carvajal-Figueroa, Cristian Archivaldo-García, Carlos Pique-Aragón, Sergio Landa-Juárez y Gerardo Izundegui-Ordóñez

Departamento de Cirugía Pediátrica, Hospital Infantil Privado, Curso de Alta Especialidad Postgrado Universidad Autónoma de México, Ciudad de México, México

\section{Resumen}

Introducción: Las hernias femorales son raras en la infancia. El defecto está abajo del ligamento inguinal. La frecuencia de un diagnóstico erróneo es de hasta el 75\%. Pueden ser resueltas mediante cirugía abierta o laparoscópica. Objetivo: Comunicar el caso de un niño con hernia femoral resuelto mediante laparoscopía. Caso clínico: Varón de 5 años, con antecedente de criptorquidia bilateral resuelta a los 2 años de edad. Padecimiento actual con 1 mes de evolución, con aumento de volumen en el tercio externo de la ingle. El ultrasonido reportó un defecto aponeurótico junto a los vasos femorales derechos. Abordaje laparoscópico, con hallazgos de defecto por abajo del ligamento inguinal. Sin recidiva a los 12 meses de seguimiento. Discusión: Las hernias femorales son raras en los niños y su diagnóstico es difícil. La exploración laparoscópica permitió identificarla al encontrar un defecto por abajo del ligamento inguinal y junto a los vasos femorales independiente del anillo inguinal profundo y de la fascia transversal.

Palabras Clave: Hernia femoral. Laparoscopía. Pediatría.

\begin{abstract}
Introduction: Femoral hernias are rare in children. The defect is below the inguinal ligament. The frequency of a misdiagnosis is up to $75 \%$. They can be resolved by open or laparoscopic surgery. Objective: To report the case of a child with femoral hernia, resolved by laparoscopy. Case report: Male, 5 years old, with a history of bilateral cryptorchidism resolved at 2 years of age. Current condition with a month of evolution with an increase in volume in the outer third of the groin. Ultrasound reported aponeurotic defect along with the right femoral vessels. Laparoscopic approach, with defect findings below the inguinal ligament. No recurrence at 12 months follow-up. Discussion: Femoral hernias are rare in children, their diagnosis is difficult. Laparoscopic examination allowed its identification, finding a defect below the inguinal ligament and adjacent to the femoral vessels independent of the deep inguinal ring and the transversalis fascia.
\end{abstract}

Key Words: Femoral hernia. Laparoscopy. Pediatrics.

\footnotetext{
Correspondencia:

*Carlos García-Hernández

Circuito Valle Dorado, 12

Col. Loma de Valle Escondido

Fecha de recepción: 18-09-2019

Cir Cir. 2020;88(S1):74-78

C.P. 52930, Atizapán de Zaragoza, Edo. de Mex., México

E-mail: carloscirped@ hotmail.com

Fecha de aceptación: 16-04-2020

DOI: $10.24875 / C I R U .20001580$

Contents available at PubMed

www.cirugiaycirujanos.com

0009-7411/@ 2020 Academia Mexicana de Cirugía. Publicado por Permanyer. Este es un artículo open access bajo la licencia CC BY-NC-ND (http://creativecommons.org/licenses/by-nc-nd/4.0/).
} 


\section{Introducción}

La incidencia de las hernias inguinales en pacientes menores de 18 años se estima entre el 0.8 y el $4.4 \%$. Hasta el $95 \%$ de las hernias inguinales en los niños son indirectas y se producen debido a la falta de cierre del proceso peritoneovaginal ${ }^{2}$. La frecuencia de las hernias femorales y directas se consideraba inferior al $2 \%$, pero a partir de los abordajes laparoscópicos se considera que representan del 5 al $7 \%{ }^{3}$. Las hernias femorales son raras en los niños y su diagnóstico es difícil, ya que con frecuencia se confunden con hernias inguinales indirectas ${ }^{2}$. Su frecuencia oscila entre el 1 y el $2 \%$ de todas las hernias en los niños ${ }^{4}$. Antes de la era laparoscópica, las hernias atípicas, entre las que se encuentran las femorales, no eran reconocidas de forma inicial. El diagnóstico no se establecía hasta que había recurrencia de la hernia y después de una extensa exploración durante una nueva operación ${ }^{4}$.

El abordaje laparoscópico permite distinguir el tipo de hernia antes de iniciar la disección y de esta forma se puede establecer el tratamiento adecuado ${ }^{2}$. Esto se debe a que las referencias anatómicas son claras: en el caso de las hernias femorales, el defecto se encuentra por abajo del ligamento inguinal y medial a los vasos iliacos². A diferencia de las hernias indirectas, las hernias femorales se presentan con igual frecuencia en ambos sexos, y son más frecuentes en el lado derecho ${ }^{4-6}$. Otra característica de estas hernias es que se presentan en su forma clásica como una tumoración no reducible lateral al tubérculo púbico, por fuera del canal inguinal, que a menudo se confunde con adenitis inguinal ${ }^{7}$. Debido a estos aspectos, la frecuencia de un diagnóstico erróneo llega hasta el $75 \%{ }^{8}$. Con una fuerte sospecha clínica, el ultrasonido es el auxiliar diagnóstico de elección $n^{9,10}$.

Existen diferentes reportes de las técnicas de reparación de estas hernias, que varían desde el abordaje abierto con disección y ligadura del saco herniario mediante abordaje inguinal o crural, hasta la reparación laparoscópica con colocación de una malla para cubrir el canal femoral ${ }^{11}$.

El objetivo de este trabajo es comunicar el caso de un paciente pediátrico con una hernia femoral, su metodología diagnóstica, los hallazgos operatorios para establecer un diagnóstico adecuado, y su resolución mediante cirugía de mínima invasión.

\section{Caso clínico}

Se trata de un paciente se sexo masculino, de 5 años, con antecedente de haber padecido criptorquidia

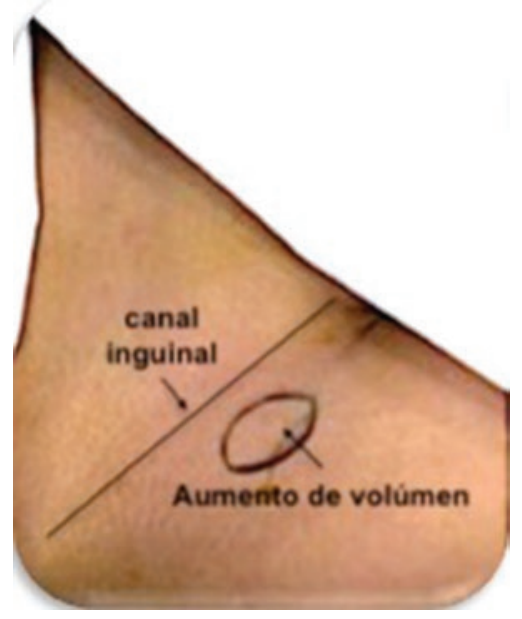

Figura 1. Aumento de volumen no reducible en la ingle por fuera del canal inguinal.

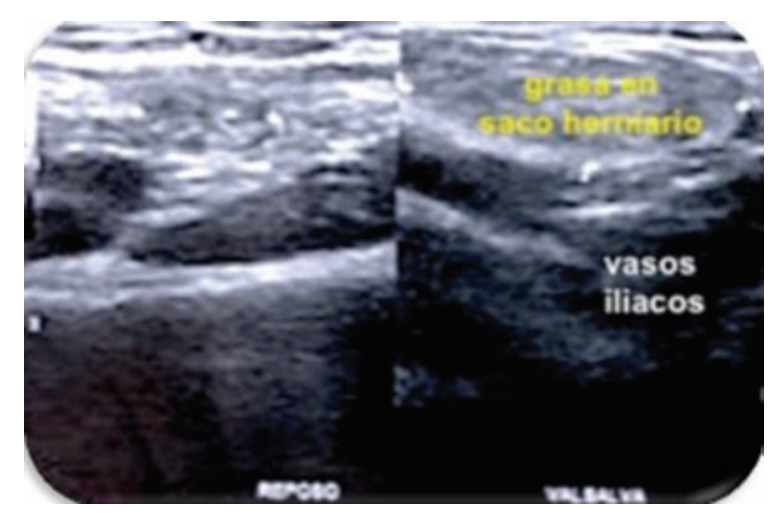

Figura 2. La ultrasonografía identifica la protrusión de grasa en un defecto junto a los vasos iliacos.

bilateral resuelta a los 2 años mediante abordaje abierto.

El padecimiento actual es de 1 mes de evolución, caracterizado por un incremento de volumen no reducible en el tercio externo de la ingle derecha, por fuera del canal inguinal, que aumenta con el esfuerzo, presentando dolor a la palpación (Fig. 1).

Se practicó ultrasonido con un transductor lineal de $9 \mathrm{MHz}$ que confirmó la presencia de un defecto aponeurótico, por el que protruye material graso que aumenta durante la maniobra de Valsalva, a un lado de los vasos femorales derechos (Fig. 2).

La resolución quirúrgica fue mediante abordaje laparoscópico de tres puertos: óptica de $5 \mathrm{~mm}$ y $30^{\circ}$ umbilical y dos puertos de 5 y $3 \mathrm{~mm}$ colocados a nivel de la cicatriz umbilical a la derecha e izquierda de la parte externa del musculo recto anterior del abdomen. Se insufló con neumoperitoneo de 8 


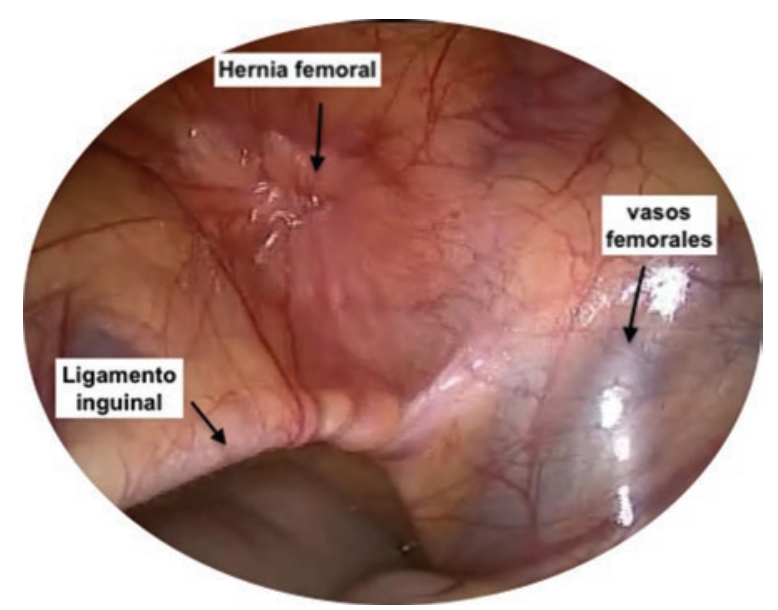

Figura 3. Vista laparoscópica en la que se aprecia la protrusión de grasa y su relación con los vasos femorales.

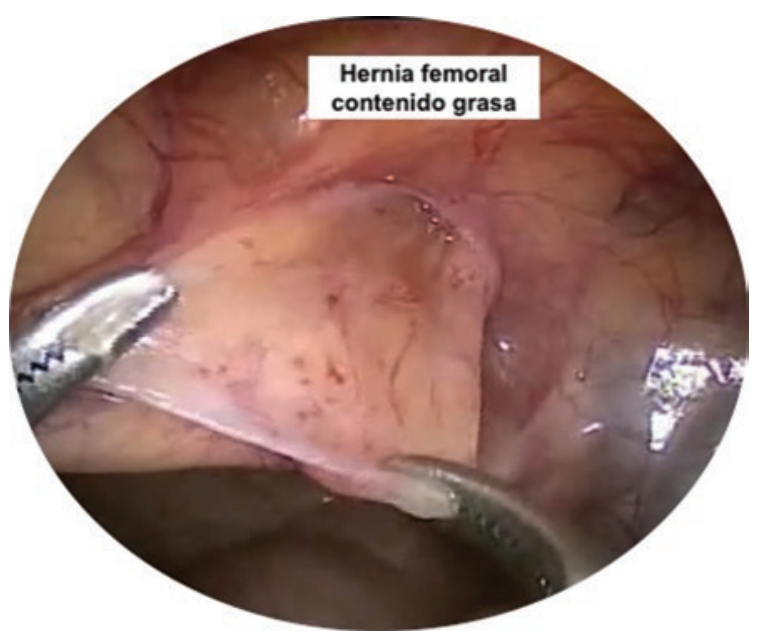

Figura 4. Hernia femoral con su contenido graso.

$\mathrm{mmHg}$ a una velocidad de $1 \mathrm{l} / \mathrm{min}$. Se exploró el canal inguinal derecho, con hallazgo de anillo inguinal interno cerrado y protrusión de tejido graso por abajo del ligamento inguinal y a un lado de la vejiga y los vasos femorales (Figs. 3 a 5). Se realizó colgajo de peritoneo hasta reducir el contenido graso de la hernia, identificando un orificio de $2 \mathrm{~cm}$, lateral a la vejiga y medial a los vasos iliacos derechos, por abajo del ligamento inguinal (Figs. 6 y 7). Se continuó la disección del colgajo peritoneal hasta identificar el pubis, así como el conducto deferente y los vasos espermáticos, y se encontró el anillo inguinal profundo cerrado. Se introdujo una malla de $5 \times 5$ $\mathrm{cm}$ (Premilene ${ }^{\circledR}$ Mesh B Braun) que se fija al pubis y las paredes anterior y lateral del abdomen con engrapadora endoscópica (Securestrap ${ }^{\circledR}$ Ethicon)

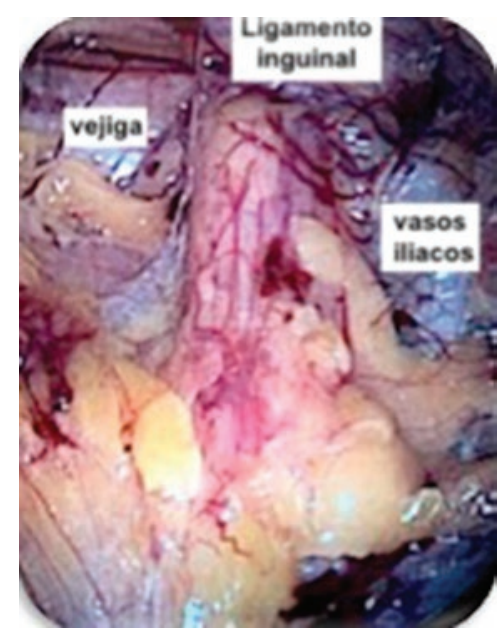

Figura 5. Orificio herniario, por abajo del ligamento inguinal, hacia la línea media del pubis y la vejiga. En la parte externa los vasos iliacos, que se convierten en femorales.

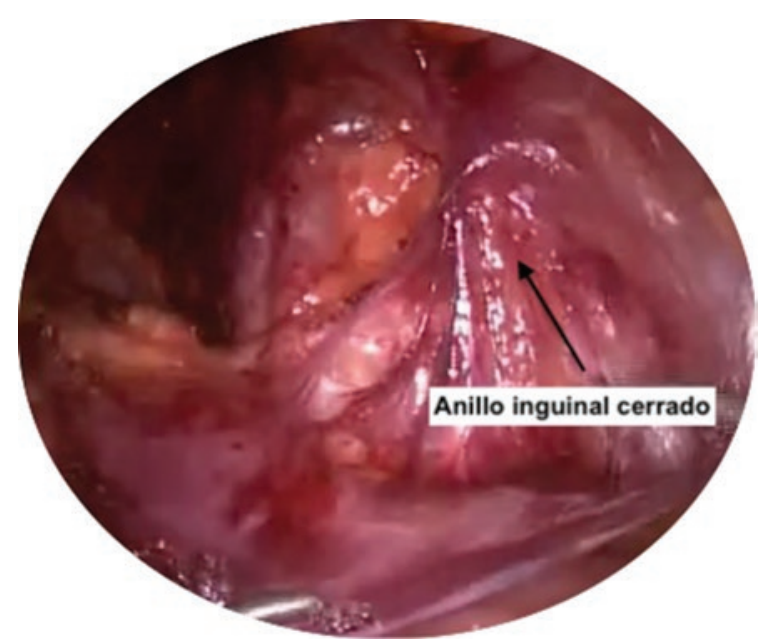

Figura 6. Vista laparoscópica que demuestra la presencia del anillo inguinal profundo cerrado.

(Fig. 8). La malla se cubrió con el colgajo peritoneal (Fig. 9). El tiempo quirúrgico fue de 40 minutos. No hubo complicaciones transoperatorias y se logró la curación de la hernia sin recidiva en un seguimiento de 12 meses.

\section{Discusión}

Las hernias femorales son raras en los niños y difíciles de diagnosticar, ya que con frecuencia se confunden con otro tipo de hernias. Se le estima una frecuencia del 1 al $2 \%$ de las hernias en la edad pediátrica ${ }^{2-4}$. Se desconoce su etiología, aunque la hipótesis más aceptada es la propuesta por McVay y 


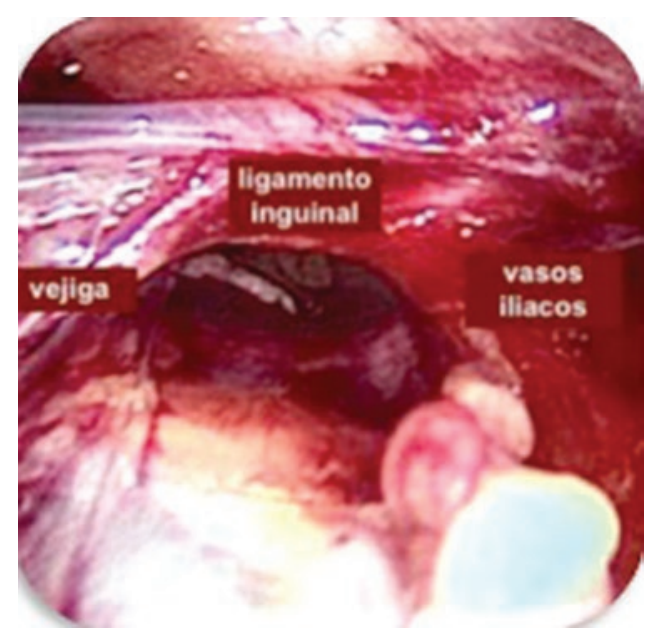

Figura 7. Orificio herniario despues de reducida la grasa. Hacia la parte medial la vegija y hacia la parte externa los vasos femorales.

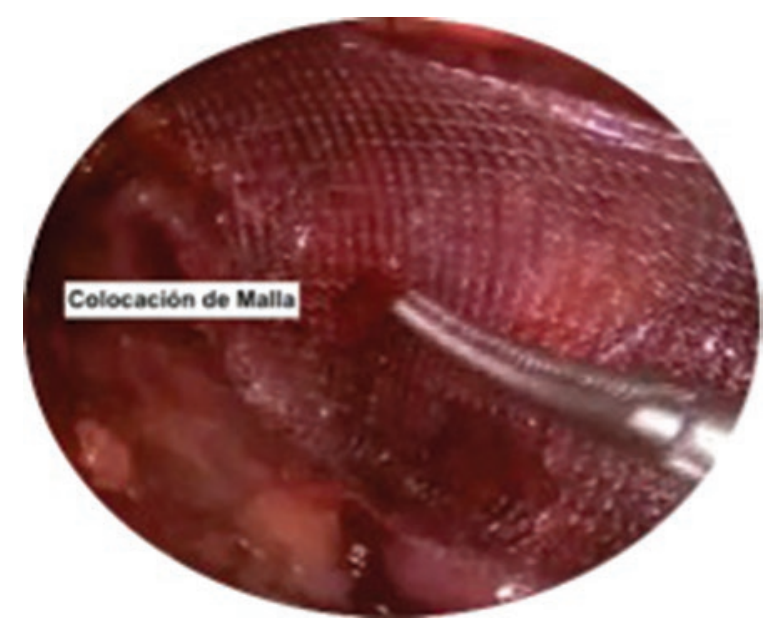

Figura 8. Colocación de una malla que se fija al piso del canal inguinal, cubriendo el orificio femoral.

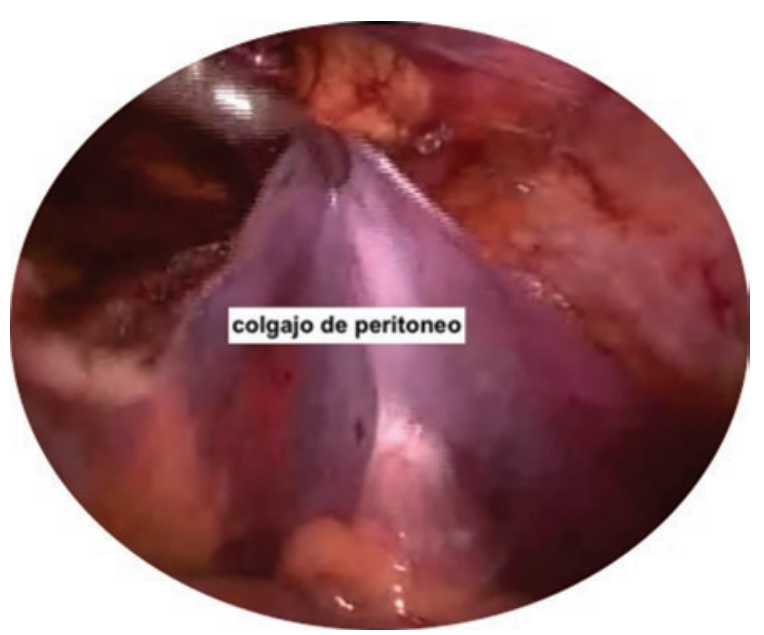

Figura 9. Colgajo peritoneal que cubre la malla.
Savage ${ }^{12}$, que refieren que una inserción estrecha de la pared posterior del canal inguinal en el ligamento de Cooper resulta en un anillo femoral de mayor tamaño. El diagnóstico preoperatorio es difícil; en el caso aquí comentado, la presencia de una tumoración por fuera del canal inguinal hizo sospechar que no se tratara de una hernia inguinal indirecta. Por esta razón se practicó un ultrasonido, el cual fue compatible con una hernia femoral debido a su contenido y a su localización medial a los vasos femorales; además, permitió excluir la posibilidad de que se tratara de una linfadenitis inguinal y demostró el defecto tal como se ha descrito ${ }^{10}$. Las exploraciones inguinales previas se han propuesto como un factor de riesgo para el desarrollo de hernias femorales; sin embargo, no han encontrado un soporte científico adecuado y más bien el antecedente podría deberse a que fue una exploración inguinal fallida ${ }^{5,8,9}$. Este paciente había sido operado de una orquidopexia bilateral, la cual no tuvo relación alguna con la hernia femoral. Estos casos pueden ser tratados mediante abordaje abierto o laparoscópico ${ }^{3}$. Nosotros sistemáticamente realizamos un abordaje por mínima invasión de la patología inguinal en los niños ${ }^{13}$, razón por la que decidimos tratar a este paciente con dicho abordaje. Además, la laparoscopía aumenta la certeza diagnóstica y permite realizar un tratamiento adecuado ${ }^{14}$ como sucedió con nuestro paciente, en quien se pudo identificar con claridad la anatomía del canal inguinal y diagnosticar el tipo de hernia al encontrarse un defecto por abajo del ligamento inguinal y junto a los vasos femorales, independiente del anillo inguinal profundo.

Las opciones terapéuticas para tratar las hernias femorales por abordaje laparoscópico son la técnica de McVay modificada por laparoscopía, en la cual el tendón conjunto y el ligamento de Cooper se usan para cerrar el canal femoral con reconstrucción posterior del peritoneo o bien con el uso de una malla de polipropileno con colgajo peritoneal, que fue la técnica utilizada por nosotros, que genera menos tensión en el orificio femoral ${ }^{14}$.

La hernia femoral es una patología rara en la edad pediátrica y es difícil de diagnosticar. El abordaje laparoscópico permite una mejor visión de la región inguinal y mejora la certeza diagnóstica. Además, hace posible la corrección de todos los tipos de defectos de la región inguinal. Los cirujanos pediatras siempre debemos considerar la posibilidad de las hernias femorales como diagnóstico diferencial en los niños con hernia. 


\section{Conflicto de intereses}

El autor declara no tener conflicto de intereses.

\section{Financiamiento}

No se recibió ningún financiamiento para realizar este trabajo.

\section{Responsabilidades éticas}

Protección de personas y animales. Los autores declaran que para esta investigación no se han realizado experimentos en seres humanos ni en animales.

Confidencialidad de los datos. Los autores declaran que han seguido los protocolos de su centro de trabajo sobre la publicación de datos de pacientes.

Derecho a la privacidad y consentimiento informado. Los autores han obtenido el consentimiento informado de los pacientes y/o sujetos referidos en el artículo. Este documento obra en poder del autor de correspondencia.

\section{Bibliografía}

1. Miltenburg DM, Nuchtern JG, Jaksic T, Kozinetiz C, Brandt ML. Laparoscopic evaluation of the pediatric inguinal hernia - a meta-analysis. $J$ Pediatr Surg. 1998;33:874-9.

2. Gödeke J, Muensterer OJ. Femoral, direct, and rare inguinal hernias in children - an update. Eur J Pediatr Surg. 2017;27:484-94.

3. Esposito C, Montinaro L, Aicchio F, Scermino S, Basile A, Armenise T, et al. Technical standardization of laparoscopic herniorraphy in pediatric patients. World J Surg. 2009;33;1846-50.

4. Schier F, Klizaite J. Rare inguinal hernia forms in children. Pediatr Surg Int. 2004;20:748-52.

5. Al-Shanafey S, Giacomantonio M. Femoral hernia in children. J Pediatr Surg. 1999;34:1104-6.

6. Gul Y. Femoral hernia in children. Ir Med J. 2000;93:183-4

7. Radcliffe G, Stringer MD. Reappraisal of femoral hernia in children. $\mathrm{Br} \mathrm{J}$ Surg. 1997;84:58-60.

8. Wright JE. Femoral hernia in childhood. Pediatr Surg Int. 1994:9:167-9.

9. Chapman $\mathrm{WHH}$, Barcia PJ. Femoral hernia in children: an infrequent problem revisited. Mil Med. 1991;156:631-3.

10. Robinson P, Hensor E, Lansdown MJ, Ambrose S, Chapman AH. Inguinofemoral hernia: accuracy of sonography in patients with indeterminate clinical features. Am J Roentgenol. 2006;187:1168-78.

11. Ceran C, Köylüoglu G, Sönmez K. Femoral hernia repair with mesh-plug in children. J Pediatr Surg. 2002;37:1456-8.

12. McVay CB, Savage LE. Etiology of femoral hernia. Ann Surg. 1961; 154:25-32.

13. García-Hernández C, Carvajal-Figueroa L, Suárez-Gutiérrez R, Landa-Juárez S. Laparoscopic approach for inguinal hernia in children: resection without suture. J Pediatr Surg. 2012;47:2093-5.

14. Aneiros BC, Cano NI, García VA, López DM, Benavent Gordo MI, Gómez Fraile A. Pediatric femoral hernia in the laparoscopic era. Asian J Endosc Surg. 2018;11:233-7. 